\title{
A Lógica Compositiva dos Artefatos Metálicos da Arquitetura Eclética de Pelotas
} The Logic of Composition in Metallic Artifacts of Pelotas' Eclectic Architecture

\author{
Ana Paula Dametto \\ Universidade Federal de Pelotas, Brasil \\ anapauladametto@hotmail.com \\ Adriane Borda Almeida da Silva \\ Universidade Federal de Pelotas, Brasil \\ adribord@hotmail.com
}

\author{
Janice de Freitas Pires \\ Universidade Federal de Pelotas, Brasil \\ janicefpires@hotmail.com \\ Luisa dalla Vecchia \\ Universidade Federal de Pelotas, Brasil \\ luisafelixd@gmail.com@hotmail.com
}

\begin{abstract}
This paper describes a method of analysis of metallic artifacts present in the eclectic buildings considered cultural heritage in the city of Pelotas. This kind of analysis is configured as a reference in order to structure didactic content to be used in courses which discuss the geometry of form in a context of architectural training. These analyses demonstrate the potential of identifying basic concepts of geometry which can be viewed in a concrete manner in the compositions being analyzed.
\end{abstract}

Keywords: Metallic artifacts of Pelotas; Analysis of geometric composition; Eclectic architecture; Didactic activities.

\section{Introdução}

A cidade de Pelotas, RS/ Brasil, apresenta um acervo urbano significativo de componentes arquiteturais metálicos vinculados às edificações ecléticas, patrimônio cultural da cidade. Em Dametto, 2009, foi realizado um inventário dos artefatos metálicos das fachadas públicas desta arquitetura histórica, com o objetivo de compreender a manifestação destes elementos. Em tal estudo, desenvolveu-se um método de inventário para a catalogação destes bens integrados.

Os componentes arquiteturais estudados foram classificados, num primeiro momento, segundo sua função. Identificaram-se dezesseis tipos de artefatos metálicos que aparecem com uma frequência de duas ou mais vezes no conjunto arquitetônico analisado. Os tipos identificados: balcão, bandeira, grade em bandeira, grade em janela de porão, grade em janela, grade em platibanda, grade em porta de madeira, gradil, guarda-corpo, janela, luminária, marquise, montante, peitoril, porta e portão. A análise da linguagem visual dos componentes arquiteturais metálicos foi realizada sob três abordagens: Aspecto Geral da Forma, observando-se os tipos de linhas que definem cada elemento (Wong,2007); Princípios de Ordem (Ching, 1998) e Glossário dos Elementos Compositivos (Dametto, 2009). Esta análise auxiliou na identificação das principais influências estilísticas nas composições dos artefatos metálicos no período de 1870 a 1931.

Conforme já relatado em Borda, Pires e Vasconcelos, 2012, um conjunto de conceitos que suporta análises sobre o ponto de vista da geometria está sendo sistematizado junto ao contexto que se insere este trabalho. Tem-se investido no desenho didático de atividades que integrem, desde os estágios iniciais de formação em arquitetura, os conteúdos de geometria, gráfica digital e projeto. Em Borda, Pires e Alves, 2012, apresentou-se um estudo que adota, como objeto de aprendizagem de geometria, elementos relativos ao patrimônio da arquitetura de Pelotas. No referido trabalho estabeleceu-se um método de análise para os ladrilhos hidráulicos presentes em Casarões da cidade, tombados pelo IPHAN. Para as análises fundamentou-se, por um lado, nos conceitos de simetria trazidos de Sanz e Moratalla, 1999. Por outro, em processos dinâmicos, de representação gráfica digital, apresentados por Celani, 2003. Desta maneira, buscando agregar mais um componente ao contexto didático, o patrimônio como referencial para processos criativos de arquitetura.

Seguindo-se com este mesmo propósito, este trabalho busca promover a construção de um conhecimento mais específico sobre os artefatos metálicos, relativos também à arquitetura pelotense. Considerou-se a possibilidade de, sob a sistematização de conteúdos de geometria apresentada em Borda, Pires e Vasconcelos, 2012, ampliar a análise formal estabelecida em Dametto, 2009.

Entende-se que este tipo de estudo adquire importância frente às práticas de documentação arquitetônica de bens de interesse cultural, preservando a memória social. Nesta perspectiva, este trabalho dá prosseguimento à Dametto, 2009, na construção de conhecimento sobre os artefatos metálicos. Sendo que se enfatiza aqui o enfoque de interesse didático.

A geometria se configura como um parâmetro objetivo para a análise da forma. Logicamente, não sustenta a complexidade de 
processos projetuais. Entretanto, permite aproximar os estudantes iniciantes de arquitetura a, pelo menos, uma das estratégias que compõem esta complexidade. Considera-se que este tipo de postura didática, de instrumentalização a partir da geometria, vai de encontro ao que autores como Silva (2001) e Wertheiner (1945) consideram. Ambos destacam a importância da reflexão sobre os processos projetuais, da caracterização dos problemas de projeto e do estabelecimento de processos conscientes de criação.

Entende-se que a compreensão da lógica compositiva a partir da estrutura geométrica da forma possa desmistificar algo da criatividade projetual, tratando-a como uma habilidade que pode ser apreendida pelo estudante.

\section{Metodologia}

Neste trabalho registra-se então a elaboração de um desenho didático para promover especificamente a ampliação de conhecimento sobre os artefatos metálicos presentes na arquitetura pelotense.

Seguindo o método estabelecido em Borda, Pires e Vasconselos, 2012, desenvolve-se uma análise sobre um elemento específico: uma janela tipo bandeira, reconhecida como elemento em ferro mais frequente nos casarões estudados em Dametto, 2009.

A partir da análise deste único elemento pretende-se que o método seja utilizado pelos estudantes, aplicado ao conjunto de elementos documentados, desencadeando um processo de construção de conhecimento sobre tais artefatos.

O trabalho foi desenvolvido através das seguintes etapas:

\section{Revisão}

Partiu-se então da sequência didática proposta em Borda et al, 2012, a qual está fundamentada em diversos autores: Carvalho (2011) para apoiar o processo de identificação e classificação de Entes Geométricos; Carvalho (2011), para a compreensão do conceito de Concordância; Ching (1998), Doczi (1990) e Elam (2001), para o de proporção; Sainz e Moratalla (1999), para simetrias; Celani (2003), para o de recursão.

\section{Seleção de um tipo de artefato metálico do patrimônio da cidade de Pelotas}

Conforme referido anteriormente, a janela tipo bandeira, estudada em Dametto, 2009, e ilustrada na figura 1, foi selecionada para registrar um método de análise e servir de material de apoio para a proposta didática aqui constituída.

A bandeira é um caixilho, fixo ou móvel, que se localiza na parte superior das portas ou janelas, com a finalidade de favorecer a iluminação aos ambientes, e quando móveis, a ventilação (CORONA e LEMOS, 1972). Foi muito utilizada em aberturas de edificações do século XIX, frequentemente caracterizada por uma estrutura em barras metálicas com desenhos diversificados e vidros coloridos, em maior parte no térreo das edificações.

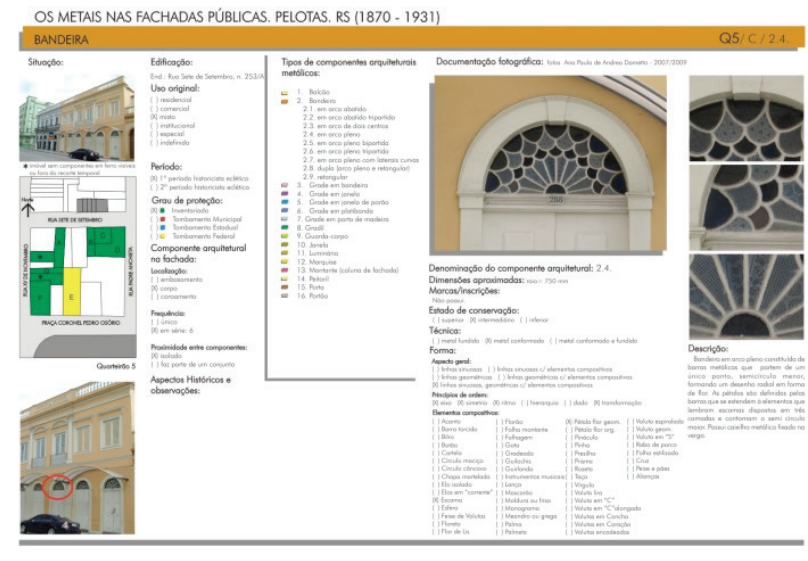

Figura 1: Ficha cadastral do elemento em ferro bandeira, adotado para análise. Fonte: Inventário "Os metais nas fachadas públicas. Pelotas. RS (1870-1931)" DAMETTO, 2009.

Dametto (2009) identificou nove tipos de bandeiras inseridas em exemplares da arquitetura pelotense. Estes tipos foram classificados segundo o contorno geral da forma, tendo sido observado que o contorno em arco pleno é o de maior recorrência. A bandeira selecionada possui contorno geral em arco pleno e encontra-se inserida no térreo do casarão localizado a Rua Sete de Setembro, n. 253a, ilustrado também na figura 1. Este edifício pertence ao primeiro período historicista eclético, o que para Pelotas corresponde às últimas décadas do século XIX.

\section{Desenho de atividades de análise}

O elemento selecionado foi analisado a partir dos conceitos geométricos anteriormente referidos.

A análise sobre os entes geométricos que compõem a bandeira estudada está ilustrada na figura 2. Demonstra-se a presença dos seguintes elementos: segmentos de retas; arcos de circunferência com ângulo de $180^{\circ}$; e arcos de circunferência com ângulos menores que este. Estes últimos formam também arcos do tipo ogival. Também se identifica a localização de cada um dos centros dos arcos, gerando-se uma infraestrutura para as análises posteriores.

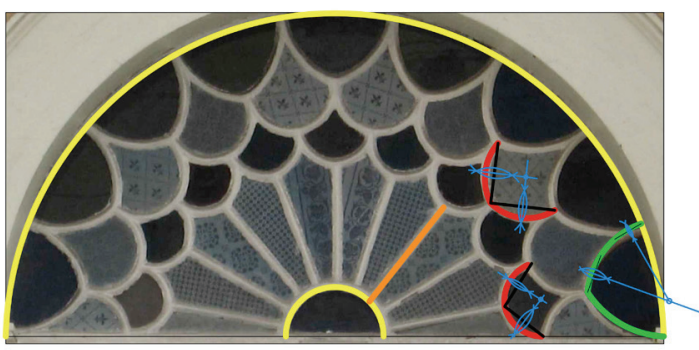

SEGMENTIS DE RETA

ARLIS DE CIRCUNFERÊNCIA DE $180^{\circ}$

ARCIS DE CIRCUNFERÊNCIA

ARCD DGIVAL

Figura 2: Identificação dos entes geométricos da janela analisada. Fonte: autores, 2013 
A sequência de análise propõe observar a forma sob o conceito de concordância, o qual, neste caso, não se faz pertinente. Os arcos de circunferência presentes na composição não concordam entre si e nem são tangentes aos segmentos de reta.

Conforme ilustrado na primeira linha da figura 3, demonstra-se o traçado para a identificação do módulo elementar da composição da janela. Formado a partir do raio do arco pleno que the configura, o qual se encontra repetido formando 18 módulos básicos. Na segunda linha desta mesma figura está lançada uma hipótese de identificação de outro módulo. Neste caso gerado a partir da reflexão do módulo básico sobre o eixo formado pelo raio do arco pleno. Sob esta leitura, ficam configurados oito módulos desse tipo, dispostos por simetria cíclica. Além de mais dois iguais ao módulo anterior, podendo-se considera-los dispostos por simetria de reflexão.
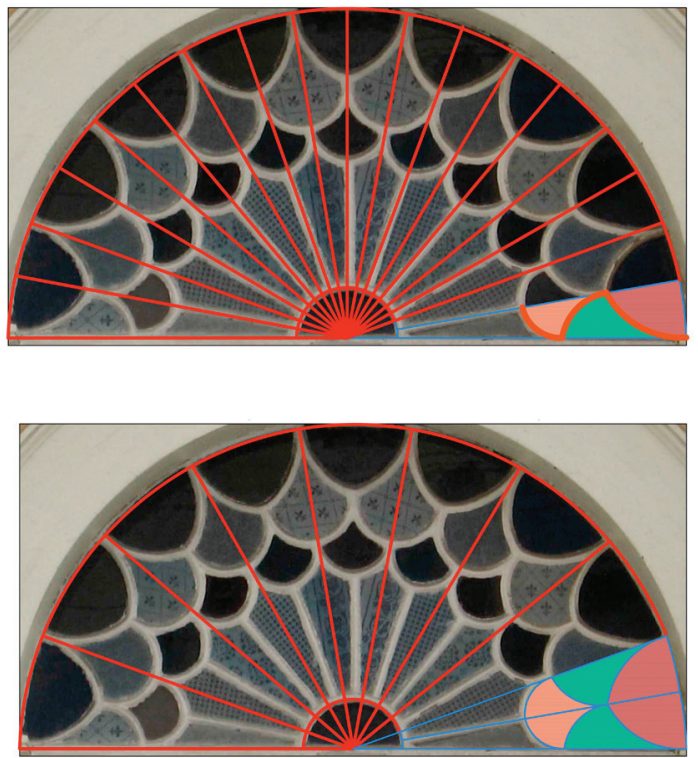

Figura 3: Na primeira linha, a Identificação do módulo básico que compõem a janela analisada; Na segunda linha, a Identificação do módulo formado pela reflexão do módulo básico. Fonte: autores, 2013.

Os traçados da figura 4 detalham as regras dos arcos que foram identificados nos dois tipos de módulos considerados como hipótese da composição: amplitude dos arcos e localização de seus centros.

A figura 5 demonstra os traçados para investigar sobre possíveis relações dimensionais entre as partes do módulo básico da composição. Foi identificada a existência da proporção raiz de dois, definida pela razão entre a soma dos raios $A$ e $B$ com o raio $B$.

A figura 6 ilustra um dos traçados realizados para investigar sobre o uso do conceito de recursão. Foi possível associar o desenho da bandeira com o tipo de recursão com adição de elementos em escala maior, aplicada de maneira não uniforme. Esta recursão obedece também um movimento cíclico em torno de um ponto central.
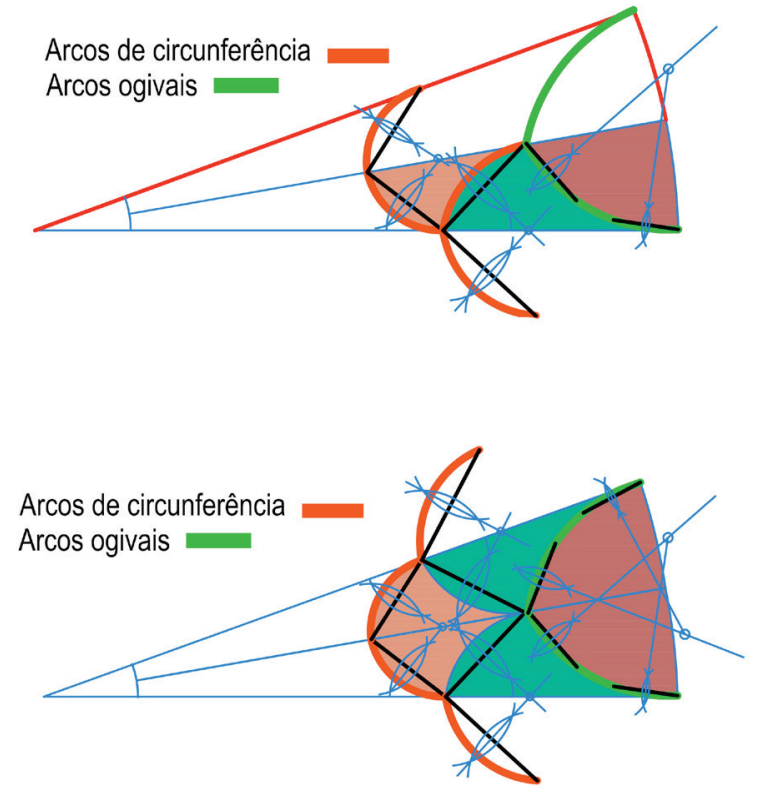

Figura 4: Identificação dos entes geométricos componentes dos módulos fundamentais da janela analisada. Fonte: autores, 2013

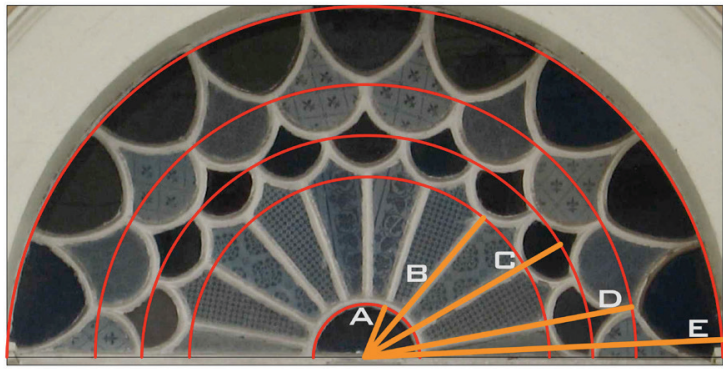

$A+B / B=1,41=\sqrt{ } 2$

Figura 5: Identificação da relação de proporção entre as partes da composição. Fonte: autores, 2013.

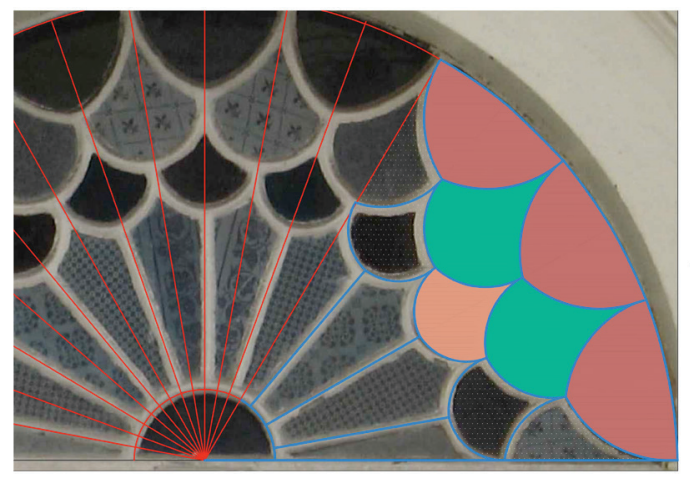

Figura 6: Identificação da regra de recursão com adição e rotação de elementos em torno de um ponto central. Fonte: autores, 2013 


\section{Resultados}

As análises registradas exemplificam concretamente os traçados que permitem evidenciar tipos de conceitos geométricos que podem explicar a composição estudada. Com isto constitui-se um material didático que pode orientar os estudantes a particularizar cada conceito, como ferramentas para constituir leituras diferenciadas sobre uma mesma forma. E, principalmente, estes estudos se configuram como construção de conhecimento sobre tais elementos. Entretanto, será o conjunto de análises sobre os artefatos em ferro da arquitetura pelotense que fornecerá subsídios para compreender a lógica compositiva de cada um deles. Neste momento, os resultados se restringem à exemplificação do emprego do método.

\section{Considerações Finais}

O tipo de análise desenvolvido no âmbito deste estudo se configura como referencial para a estruturação de práticas didáticas. Especialmente para serem inseridas em disciplinas que tratam da geometria da forma em contextos de formação em arquitetura.

No contexto em questão, estes estudos específicos sobre a lógica compositiva de artefatos metálicos da arquitetura pelotense passam a ser empregados sob a forma de exercícios didáticos. Passam a ser utilizados a partir deste segundo semestre de 2013, junto à disciplina de Geometria e Gráfica Digital I, da Faculdade de Arquitetura e Urbanismo/ UFPel.

Deve-se destacar que este trabalho já está sendo ampliado, agregando-se conceitos de lógica, sistematizados em Mitchell (2008). Entende-se que a base conceitual de geometria aqui estabelecida resulta suficiente para passar à fase de formalização da lógica compositiva dos elementos a serem analisados.

\section{Referências}

Borda, A. Pires, J. F. Vasconselos, T. B. (2012). O Desenho Didático para o Insight. In: XVI Sigradi - Congresso da Sociedade Ibero Americana de Gráfica Digital: Forma(in)formação. Fortaleza: Expressão Gráfica e Editora. v.01. p.277- 280

Borda, A. B. A. S, Pires, J. F., Alves, C. M. (2012). A Lógica Compositiva dos Ladrilhos Hidraúlicos nos Casarões de Pelotas. In: IV Congreso Internacional de Expresión Gráfica e IX Congreso Nacional de Profesores de Expresión Gráfica. Gráfica del Diseño:tradición e innovaciones. La Plata: Color Magenta Gráfica, v.01. p.41 - 44

Carvalho, B. A. (1967). Desenho Geométrico. Rio de Janeiro: Imperial Novo Milênio.

Celani, G. (2003). Cad Criativo. Rio de Janeiro: Editora Campus.

Ching, F. D. K. (1998). Forma espaço e Ordem. São Paulo: Martins Fontes.

Doczi, G. (1990) O poder dos limites: harmonias proporções na natureza. São Paulo: Mercuryo.

Elam, K. (2001). Geometry of design: studies in proportion and composition. New York: Princeton Architectural Press.

Dametto, A. A. (2009). Os metais no patrimônio arquitetônico urbano de Pelotas, RS - 1870 a 1931. Dissertação. Programa de Pós-graduação em Memória Social e Patrimônio Cultural. Universidade Federal de Pelotas, Brasil.

Mitchell, W. J. (2008). A Lógica da Arquitetura. Projeto, Computação e Cognição. Campinas: Editora Unicamp.

Sanz, M. A. Moratalla, A. (1999). Simetría. Serie Geometría y Arquitectura II, Cuadernos de Apoyo a la Docencia del Instituto Juan de Herrera. Madri: Publicaciones de la Escuela Superior de Arquitectura de Madrid.

Silva, Elvan. 1984. Uma introdução ao projeto arquitetônico. Porto Alegre: Ed. da Universidade, UFRGS.

Wertheimer, Max. (1945). Productive Thinking. New York: Harper. 